\title{
Factores familiares en la recaída de pacientes esquizofrénicos*
}

\section{Réplica californiana de la investigación británica sobre emoción expresada.}

\section{Christine E. Vaughn, Karen Sorensen Snyder, Simon Jones, William B. Freeman y Ian R. H. Falloon}

\section{Resumen}

Nuestro estudio de la atmósfera emocional de los hogares de los pacientes esquizófrenicos en California del Sur ha replicado los descubrimientos británicos en relación con la influencia del ambiente familiar sobre el curso de la esquizofrenia. Como en los estudios británicos, las críticas y la implicación emocional excesiva de un familiar clave hacia el paciente en el momento de su ingreso constituyen el mejor predictor aislado de recaída sintomática en los nueve meses posteriores al alta hospitalaria. Al igual que en los resultados británicos, la asociación entre la emoción expresada de los familiares y la recaída fue independiente de todas las demás variables investigadas. Los resultados transculturales sobre los efectos preventivos de la medicación sugieren que los clínicos deben considerar la atmósfera emocional del hogar, a la hora de explicar los fracasos de la medicación.

A pesar de uso extendido de los eficaces fármacos antipsicóticos, las tasas de recaída y los reingresos de las personas que padecen esquizofrenia se mantienen preocupantemente altos. Una vez más ha aumentado el interés entre los investigadores por características ambientales que pueden influir sobre el pronóstico de los pacientes dados de alta. A lo largo de más de veinte años se ha venido desarrollando una prometedora línea de investigación por parte de los miembros del Medical Research Council (MRC) de la Unidad de Psiquiatría Social de Londres, en torno a la influencia de las relaciones familiares sobre el curso de los trastornos esquizófrenicos ya establecidos. Leff (1) ha revisado esta serie de estudios británicos realizados por Brown et al. (2-6).

De particular relevancia para nuestra réplica americana de la investigación británica sobre factores familiares, han sido los estudios de Brown et al. (5) y de Vaughn y Leff (6). Aunque fueron llevados a cabo por diferentes equipos de investigación, y con un intervalo de diez años de diferencia, estos dos estudios arrojaron resultados impresionantemente si- 
milares. Ambos concluyen que, para los pacientes que viven con sus familiares, el mejor predictor de recaída sintomática en los nueve meses después del alta del hospital, fue la emoción expresada por un familiar clave hacia el paciente durante una entrevista estandarizada, valorada con un elevado nivel de fiabilidad y administrada poco después del ingreso del paciente. El índice de emoción expresada (EE) empleado en ambos estudios tenía tres componentes: 1) el número de comentarios críticos hechos por el-familiar cuando hablaba sobre el paciente; 2 ) hostilidad (que raramente aparece en ausencia de un elevado número de comentarios críticos); y 3) una acusada implicación emocional excesiva. El índice se usó para categorizar a los pacientes en procedentes de hogares de alta EE o de baja EE. En ambos estudios, los pacientes de hogares de alta EE recayeron significativamente con más frecuencia (3,5 de 8 casos) que los de baja EE. En los dos estudios esta asociación era independiente de la desorganización conductual y del deterioro laboral previos del paciente. Sin embargo, las relaciones entre el índice de $\mathrm{EE}$, y las recaídas estaba mediatizada por el nivel de medicación del paciente y por la cantidad de contacto directo cara a cara con la familia.

Cuando Vaughn y Leff (6) consideraron los efectos aditivos de los dos factores protectores, la medicación regular y el contacto reducido, fueron capaces de predecir pautas de recaída aun más precisas que en los primeros estudios. Sus resultados sugieren que la combinación de la medicación antipsicótica regular y el contacto mínimo con los familiares altamente críticos o excesivamente implicados a nivel emocional podía reducir en gran medida la tasa de recaídas; es decir, los efectos negativos de la emoción expresada podían ser modificados mediante los efectos combinados de estos dos mecanismos protectores. Al mismo tiempo, aquellos pacientes que residían en ambientes familiares más tolerantes, y emocionalmente más neutrales (baja EE), tenían más probabilidades de mantenerse ajustados durante un período de nueve meses, tomaran o no medicación, y estuvieran o no en contacto frecuente con sus familiares.

Un seguimiento de dos años de los pacientes estudiados por Vaughn y Leff confirmó que las valoraciones de EE de los familiares eran predictivas de las recaídas durante períodos de tiempo superiores a los nueve meses después del ảlta hospitalaria (7). Asimismo, registros de las respuestas electrodérmicas de los pacientes esquizofrénicos han proporcionado una evidencia más directa del impacto de los familiares de alta y baja EE en estas personas $(8,9)$.

La consistencia de los resultados de los estudios de la Unidad de Psiquiatría Social del MRC tiene implicaciones para el tratamiento y la rehabilitación de los pacientes esquizofrénicos. El refinamiento progresivo de los métodos de investigación y la verificación de resultados en diversos estudios con períodos de tiempo cada vez mayores dan credibilidad a los hallazgos inequívocos que se han observado. Sin embargo, todos los estudios de EE hasta la fecha han utilizado muestras de pacientes de Londres; sin que se haya abordado todavía el problema de la especificidad cultural de la EE. Puede ocurrir que las variaciones culturales, étnicas y sociales en la intensidad y el impacto de la EE den lugar a diferentes niveles de línea base para familias de procedencia no británica. Los clínicos pueden mostrarse reticentes, comprensiblemente, a iniciar programas de intervención basados en la EE, antes de que la relevancia transcultural del concepto haya quedado establecida. Esta fue la razón de nuestra réplica internacional del trabajo británico.

La meta principal del presente estudio era replicar los trabajos de 
Brown et al. (5) y Vaughn y Leff (6) con una muestra de pacientes de California del Sur. Como en los estudios londinenses, se investigó la influencia de los factores sociales y familiares en el curso de la esquizofrenia, recurriendo a las mismas técnicas de entrevista estandarizada. Se utilizó un diseño similar de seguimiento prospectivo a los nueve meses, con medidas independientes de la conducta pasada, de la respuesta emocional actual de los familiares y de la presencia o ausencia de recaída subsiguiente. En este tipo de diseño transversal no se podían comprobar hipótesis sobre la etiología, pero esperábamos encontrar una asociación entre un alto grado de EE en los familiares y la recaída florida de síntomas, independientemente de otros factores, tales como la duración de la historia psiquiátrica, el tipo de síntomas o la severidad del trastorno en el momento del ingreso.

Se tomaron todo tipo de precauciones para replicar con exactitud los procedimientos utilizados en los estudios británicos originales. La participàción del psicólogo del estudio de 1976 (C. E. V.) y de dos psiquiatras entrenados en Inglaterra (S.J. e I.R.H.F.) permitió conseguir la continuidad de la investigación y ayudó a asegurar que los datos de las evaluaciones sociales y psiquiátricas en las dos culturas fueran comparables.

En este informe se presentarán los principales resultados del pronóstico a los nueve meses del estudio del Hospital Estatal de Camarillo (California)/UCLA. Los resultados del seguimiento de los pacientes a los dos años están en fase de recogida y serán publicados posteriormente.

\section{SUJETOS Y METODOS}

\section{Sujetos}

Para que un paciente participara en el estudio, debía cumplir los siguientes requisitos: 1) edad, de 17 a 50 años; 2) hospitalización en el mes anterior a la entrevista inicial; 3) origen étnico angloamericano (el trabajo piloto de los estudios británicos sugería que había variaciones considerables en los estilos de respuesta y niveles de EE según el grupo étnico. Por lo tanto no deben analizarse los datos de diferentes grupos étnicos juntos, ni generalizar los resultados de un grupo a otro. Los investigadores británicos decidieron, en consecuencia, estudiar únicamente personas de ascendencia anglosajona. Limitando nuestra muestra de pacientes a angloamericanos, tratamos de reducir las variaciones subculturales, seleccionando pacientes que se equipararan al máximo a los criterios de la muestra británica); 4) vivir con uno de los padres, cónyuge u otro familiar cercano durante al menos uno de los tres meses anteriores al ingreso; y 5) que fueran esquizofrénicos de acuerdo con la definición que se expondrá más abajo.

La mayoría de nuestros sujetos procedía de las unidades de admisión de agudos del Hospital Estatal de Camarillo. En una estimación conservadora, este hospital recibe entre 80 y 100 ingresos semanales en estas unidades. No encontramos ninguna vía más rápida de selección que revisar al menos semanalmente de forma minuciosa todos los nuevos ingresos, labor que realizó el psiquiatra investigador (S. J.), ayudado por un psicólogo. Las edades y orígenes étnicos fueron recogidos a partir de las áreas de procedencia y de los historiales, tomándose nota del diagnóstico asignado al ingresar. Aproximadamente el 70 por 100 de los pacientes ingresados recibieron un diagnóstico de esquizofrenia o de trastorno esquizoafectivo. Los detalles sobre la vida del paciente antes del ingreso se obtuvieron de los informes de los asistentes sociales y posteriormente fueron confirma- 
dos con el paciente y los familiares directamente. Este contacto también ofrecía una útil oportunidad para realizar una breve evaluación diagnóstica, así como de la capacidad del paciente para participar en la entrevista diagnóstica del Examen del Estado Actual (Present State Examination, PSE en adelante). Si, al finalizar este proceso, un paciente seguía pareciendo adecuado, se concertaba una cita con él para que acudiera al Mental Health Clinical Research Center (MHCRC), donde se le administraba el PSE.

Los pacientes de Camarillo que participaron en el estudio representan menos del 20 por 100 de los pacientes ingresados con trastornos agudos en el periodo considerado. La principal razón de la exclusión era la falta de contacto con la familia durante los tres meses antes del ingreso, mientras que el origen étnico no angloamericano constituía una pequeña proporción de las exclusiones.

Los procedimientos semejantes de screening en otros tres centros de internos proporcionaron los pacientes restantes, en los que se identificó secuencialmente su adecuación al estudio.

\section{Evaluación del estado del paciente}

El estado del paciente fue evaluado en las dos semanas siguientes al ingreso mediante el PSE 10, la Escala de Evaluación Psiquiátrica (Psychiatric Assessment Scale, PAS en adelante) 11 y un cuestionario sobre la historia psiquiátrica y social. El diagnóstico se realizaba por el sistema de clasificación CATEGO. La mayoría de los pacientes $(\mathrm{N}=65)$ fueron clasificados como «psicosis esquizof rénica» (clase $S$ ), con cuatro pacientes en la clase $\mathrm{P}_{-\ll p s i c o s i s}$ paranoide». En los casos en los que existía alguna posibilidad de que el trastorno primario fuera de tipo afectivo, de origen orgánico, o secundario al alcohol o al abuso de otras drogas, el paciente era excluido del estudio.

La selección diagnóstica para el estudio fue llevada a cabo por tres clínicos, que administraban y valoraban el PSE. Se hicieron cálculos de fiabilidad a intervalos regulares durante el proceso de selección de la muestra, valorando conjuntamente entrevistas filmadas en vídeo. El acuerdo exacto ítem por ítem para el PSE completo, oscilaba entre el 80 y el 90 por 100. El acuerdo mínimo sobre síntomas esquizofrénicos fue del 90 por 100 , mientras que el acuerdo sobre la presencia o ausencia de síntomas schneiderianos alcanźó el 100 por 100.

Las entrevistas del status mental actual (PSE y PAS) fueron repetidas en el momento del alta del hospital. La duración media de las hospitalizaciones fue de 26 días. En aquellos casos en los que un paciente abandonara el hospital a la semana del ingreso se hizo únicamente la evaluación inicial.

\section{Evaluación de los familiares}

Paralelamente a la evaluación psiquiátrica inicial del paciente, y tras obtener el consentimiento formal, se administró la versión abreviada de la Entrevista Familiar de Camberwell (Camberwell Family Interview, CFI en adelante) 12 al (los) familiar (es) clave (s) del paciente, por un miembro del equipo de investigación de factores familiares (W. B. F., K. S. S., y C. E. V.). Una descripción detallada de la CFI y su desarrollo puede encontrarse en otra parte 13,14 . De forma resumida, se trata de una entre- 
vista en profundidad semiestructurada, que en su formato abreviado tiene una duración aproximada de hora y media. Se trata de suscitar un informe de las circunstancias del ambiente en el hogar en los tres meses precedentes al ingreso del paciente, en especial sobre los detalles de la aparición del episodio actual, y del impacto del mismo en varios aspectos de la vida. familiar. Al mismo tiempo se observa la conducta del familiar en la situación de entrevista y se toma nota de los sentimientos expresados sobre los miembros de la familia, especialmente el paciente. Se tienen en cuenta el autoinforme de las emociones y la expresión espontánea de sentimientos durante la entrevista, aunque el énfasis se pone en este último aspecto. Todas las entrevistas son grabadas magnetofónicamente para su análisis posterior.

Los entrevistadores fueron entrenados por uno de nosotros (C. E. V.) durante varios meses en el uso de la CFI y de la valoración de las escalas de EE, utilizando grabaciones de entrevistas de los estudios británicos. Se alcanzaron altos niveles de fiabilidad interjueces con las valoraciones ori-. ginales (correlación de Pearson mínima de 0,80). A lo largo del estudiō, se valoraron conjuntamente grabaciones de entrevistas seleccionadas al azar con objeto de asegurar niveles aceptables de fiabilidad. Como en los estudios británicos, se vio en todos los casos al cónyuge de los pacientes casados. En los casos de pacientes solteros que vivían con ambos padres, la madre y el padre fueron entrevistados separadamente. De un total de 41 familias en las que se mantenían ambos padres, en 36 de ellas se entrevistó a los dos. En las cinco restantes, solo se vio a uno de los padres (en cada uno de ellos el padre entrevistado era alto en EE). Veintidós pacientes procedían de hogares con uno solo de los padres; en tres de estos pacientes, el padre ausente fue también entrevistado, por su contacto regular con el paciente. De los seis pacientes restantes de la muestra total de 69, cuatro de ellos estaban viviendo con sus cónyuges, un paciente vivía con su hijo adulto y otro con un tutor.

Las valoraciones de la respuesta emocional se hicieron en las mismas escalas que en el estudio de Vaughn y Leff (6), enfatizando las tres escalas que componen el índice global de la EE de los familiares en el estudio británico, es decir, el número de comentarios críticos hechos acerca del paciente, la hostilidad y la implicación emocional excesiva. En otra parte puede encontrarse información detallada sobre estas y otras medidas, (13-15).

En los casos en los que los dos padres fueron entrevistados, se utilizó la mayor de las valoraciones de EE para determinar la inclusión de la familia en el subgrupo de alta o en el de baja EE.

\section{Procedimiento de seguimiento}

Los resultados de un estudio piloto revelaron que los pacientes de $\mathrm{Ca}$ lifornia tenían menos probabilidad que los británicos de ser dados de alta sin síntomas, o de recibir cuidados poshospitalarios regulares. Estas diferencias reclamaban una monitorización más estrecha del paciente después del alta, así como una mayor extensión y unos criterios de recaída más exigentes que los británicos.

Todos los pacientes incorporados al estudio de factores familiares fueron seguidos regularmente durante un período de nueve meses después del alta hospitalaria o durante un período más corto si padecían una recaída de forma clara. El progreso de cada paciente fue seguido de cerca por la secretaría de la investigación despues del alta. A través de las llamadas 
telefónicas mensuales a familiares clave se obtenía una información detallada específica, que después era comunicada al psiquiatra de la investigación (S. J.). Esta información, junto con la obtenida por el propio psiquiatra en llamadas posteriores, era utilizada para decidir si se había producido un cambio en el estado clínico.

El psiquiatra visitaba al paciente y realizaba una evaluación de seguimiento que incluía el PSE, cuando se daba alguna de las siguientes circunstancias: 1) cuando el paciente había sido reingresado en un centro psiquiátrico a causa de un incremento en la sintomatología; 2) cuando el familiar informaba de un empeoramiento significativo de los síntomas esquizofrénicos de al menos una semana de duración; 3) cuando conductas impulsivas, delirios expresados públicamente o alucinaciones, daban lugar a una perturbación social significativa o a una crisis familiar (por ej., problemas con la policía); 4) si el empeoramiento de síntomas daba origen a una visita de urgencia no programada a una clínica de salud mental, a un psiquiatra, o a un médico; 5) cuando se había producido un deterioro tal en el funcionamiento del paciente que éste tuviera que ser trasladado a un ambiente en el que recibía más cuidados (por ej., trasladarse a su casa o a la de sus padres), o que los amigos o familiares pensaran que era necesario aumentar de forma significativa el nivel de ayuda proporcionado al paciente; y 6) cuando el aumento de síntomas llega a un punto en el que sè requiere un incremento importante en la medicación del paciente.

La.evaluación del seguimiento incluye los siguientes procedimientos: a) la administración de la entrevista del PSE; b) cumplimentación de la PAS; $c$ ) recogida de los detalles sobre la medicación prescrita al paciente y su adhesión a la misma durante este período de tiempo; y d) un estimación del número de horas pasadas por el paciente con sus familiares, utilizando preguntas de la sección «Distribución del tiempo» de la CFI.

Los detalles sobre los apartados $c$ ) y $d$ ) fueron recogidos a través de una llamada telefónica al familiar clave.

Si el psiquiatra estimaba que el paciente había sufrido una recaída, no se llevaban a cabo más evaluaciones de seguimiento. Los pacientes que se consideró que no habían recaído, y todos los demás pacientes del estudio, fueron reevaluados nueve meses después de sus altas del hospital, como en los estudios británicos.

\section{Definición de las recaídas}

El equipo de investigación británico agrupó a los pacientes en las categorías de «recaída» y «no recaída». Distinguían entre dos tipos de recaída. El tipo I suponía un cambio de un estado normal o no esquizofrénico a un estado de esquizofrenia tal como lo definen los criterios diagnósticos CATEGO basados en el PSE. El tipo II suponía una marcada exacerbación de síntomas esquizofrénicos persistentes. La categoría de no recaída incluía dos tipos de pacientes: aquellos que no habían padecido síntomas esquizofrénicos. durante el seguimiento y los pacientes que habían experimentado síntomas esquizof rénicos continuamente a lo largo del seguimiento, pero que se habían mantenido estables o solamente con leves fluctuaciones.

En el presente estudio, la determinación de recaída en un paciente libre de síntomas cuando se le daba de alta del hospital, planteó pocos problemas. Là referencia a los registros de los contactos telefónicos y la comparación de los datos del PSE y de la PAS en el momento del alta y du- 
rante el seguimiento indicaban el retorno de síntomas floridos y de problemas de conducta. Sin embargo, la determinación de recaídas para aquellos pacientes que padecen síntomas de forma persistente (por ej., alucinaciones continuadas) al ser dados de alta, y que presentan los mismos síntomas durante el seguimiento, of recía algunas dificultades.

La aproximación adoptada por el MHCRC es un refinamiento posterior de los criterios clínicos utilizados por los investigadores británicos para medir las recaídas.

La evaluación de si los síntomas de un paciente habían empeorado significativamente se hacía comparando los datos de alta y del seguimiento sobre la severidad de los síntomas esquizof rénicos característicos, es decir, a) alucinaciones, $b$ ) delirios, y $c$ ) lenguaje incoherente. La PAS se mostró particularmente útil para este propósito. A diferencia del PSE, fue diseñada principalmente para reflejar cambios en la severidad de los síntomas esquizofrénicos. Las alucinaciones, delirios e incoherencia son valorados independientemente en una escala de 0 a 4 , donde catla punto está definido operacionalmente. En este estudio se obtuvo consistentemente una fiabilidad interjueces mínima de 0,85 . Se establecieron criterios para identificar incrementos en estas tres dimensiones de la PAS, como medio de definir la recaída en los pacientes cuyos síntomas habían empeorado en la evaluación de seguimiento. Tras largas discusiones, y después de la aplicación previa de varias estrategias de valoración, con una muestra de pacientes, en los que sobre la base clínica existía un claro acuerdo en que pertenecían a las categorías de recaída y no recaída, se desarrollaron los siguientes criterios para determinar «exacerbaciones significativas»:

1. Un incremento total de 3 puntos en una o más de las tres escalas, fue considerado una exacerbación significativa, con la puntualización de que un cambio de un solo punto de 0 a 1 en cualquiera de las escalas no era tenido en cuenta, cero representaba ausencia de síntomas, mientras que 1 indicaba síntomas que no eran claramente patológicos.

2. Si aparecía un cambio en una sola de las escalas era necesario un incremento de 2 puntos para considerar una exacerbación significativa, siempre que se alcanzara la pưntuación de 4 de severidad máxima de la escala. Por tanto, el aumento en la puntuación de 0 a 2 o de 1 a 3 no se consideraba exacerbación significativa.

Teniendo en cuenta la diversidad del curso y del pronóstico de la esquizofrenia, el MHCRC desarrolló seis categorías de pronóstico en términos de exacerbación-recaída/mejora-remisión continua. La operacionalización de las categorías se basó en las valoraciones del PSE y de la PAS, así como en los datos clínicos obtenidos en las llamadas telefónicas de rutina durante el período de seguimiento después del alta.

Las seis categorías de.pronóstico fueron las siguientes (estado en el alta-estado en el seguimiento): $A$, remisión-recaída; $B$, persistencia de síntomas-exacerbación; $\mathrm{C}$, remisión-remisión, $\mathrm{D}$, persistencia de síntomasmejoría; E, persistencia de síntomas-remisión; F, alta persistencia de síntomas mantenimiento de la alta persistencia de síntomas.

Las seis categorías fueron posteriormente reducidas a tres: exacerbación-recaída, A y B (recaída); mejora-remisión, C,D, y E (no recaída), y ausencia de cambios, $F$ (no valorable).

Los signos y síntomas característicos de esquizofrenia fueron valorados para cada entrevista del PSE, arrojando una puntuación total de síntomas esquizofrénicos. Las valoraciones de delirios, alucinaciones e incoherencia se obtuvieron también para cada administración de la PAS. Junto con los datos obtenidos por teléfono, estas puntuaciones numéricas fueron utili- 
zadas para tomar las decisiones finales sobre el tipo de pronóstico. Las valoraciones de las recaídas fueron hechas a ciegas por dos psiquiatras que no habían realizado los exámenes de estado mental. El acuerdo interjueces, a la hora de definir si un caso pertenecía a las categorías de no recaída, recaída y no valorables, fue del 92 por 100. Como en los estudios británicos, ninguno de los evaluadores del estado mental çonocía las valoraciones de EE de los familiares en el momento de la reevaluación del paciente.

\section{RESULTADOS}

La mayoría de los 69 pacientes que participaron en el estudio de factores familiares del MHCRC fueron hombres ( 77 por 100 , que nunca habían estado casados ( 87 por 100 ) y que vivían con uno u ambos padres en el. momento del ingreso (91 por 100). Su edad media era de 25,6 años. La muestra se diferenciaba notablemente de las muestras más equilibradas usadas por los estudios británicos en esta elevada proporción de sujetos varones, jóvenes y solteros. Por ejemplo, sólo el 29 por 100 de los 138 pacientes británicos ( $\mathrm{N}$ combinado) eran varones y solteros; la proporción correspondiente en el Sur de California era de un 72 por 100. También ciertas variables que indicaban mayor cronicidad estaban más presentes en la muestra californiana. Solamente el 17 por 100 eran pacientes de primer ingreso, comparado con el 54 por 100 del grupo británico. También era más frecuente entre los sujetos californianos que entre los pacientes británicos haber tenido 3 ingresos previos o más (55 por $100 \mathrm{v} 16$ por 100) y sufrir el primer episodio de esquizofrenia hace más de cinco años del comienźo del estudio (42 por 100 v 28 por 100). Mostraban significativamente mayor deterioro laboral y desorganización conductual en los meses anteriores al ingreso, de acuerdo con varios índices de funcionamiento social y clínico.

No se detectaron diferencias transculturales en la distribución por la clase social, o en los diagnósticos; la mayoría de los pacientes de ambos lugares recibían un diagnóstico de esquizofrenia nuclear.

\section{RECAIDA}

La tabla 1 muestra el pronóstico al final de los nueve meses del período de seguimiento, de los 69 pacientes, a partir de las seis categorías de pronóstico del MHCRC.

Solamente el 43 por 100 de la muestra total de los 69 pacientes se in: cluían en la categoría de no recaída, en comparación con el 66 por 100 de los pacientes de los estudios británicos $\left(\mathrm{X}^{2}=10,22 ; \mathrm{P}\right.$ II $\left.<0,001\right)$. La relación entre estado clínico en el momento del alta y en el del seguimiento no era significativa. De los 34 pacientes en remisión al alta (49 por 100 de la muestra), el 50 por 100 tuvo recaídas con síntomas esquizofrénicos durante el seguimiento, mientras que el 63 por 100 de los pacientes dados de alta con síntomas persistentes ( 51 por 100 de la muestra) mostraron una exacerbación de síntomas (grupo B) o no mejora (grupo F). Los 15 pacientes de la última categoría (22 por 100 de la muestra total) mostraron alta persistencia de síntomas tanto en el alta como en el seguimiento, una pauta que no se observa entre los pacientes británicos estudiados. Al igual que gran parte de la muestra más amplia, muchos de estos pacientes 
TABLA 1

Resultado del seguimiento de 69 esquizofrénicos a los nueve meses

\begin{tabular}{ccccc}
\hline $\begin{array}{c}\text { Categoría de } \\
\text { pronóstico } \\
\text { (porcentaje) }\end{array}$ & Grupo & Alta & Seguimiento & $\begin{array}{c}\text { Número } \\
\text { pacientes }\end{array}$ \\
\cline { 2 - 5 } $\begin{array}{c}\text { Recaída } \\
\text { (35 por 100) }\end{array}$ & A & Remisión & Recaída & 17 \\
\cline { 2 - 5 } & B & $\begin{array}{l}\text { Persistencia } \\
\text { de síntomas }\end{array}$ & Exacerbación & 7 \\
\hline $\begin{array}{c}\text { No recaída } \\
(43 \text { por 100) }\end{array}$ & C & Remisión & Remisión & 17 \\
\cline { 2 - 5 } & D & $\begin{array}{l}\text { Persistencia } \\
\text { de síntomas }\end{array}$ & Mejoria & 6 \\
\hline & E & $\begin{array}{l}\text { Persistencia } \\
\text { de síntomas }\end{array}$ & Remisión & 7 \\
\hline $\begin{array}{c}\text { Sin cambios } \\
(22 \text { por 100) }\end{array}$ & F & $\begin{array}{l}\text { Alta persistencia } \\
\text { de síntomas }\end{array}$ & Altà persistencia \\
\hline
\end{tabular}

TABLA 2

Puntuaciones medias de grupo de la PAS*

\begin{tabular}{|c|c|c|c|c|}
\hline \multirow[b]{2}{*}{$\begin{array}{l}\text { Categoría de } \\
\text { pronóstico }\end{array}$} & \multirow[b]{2}{*}{ Grupo } & \multicolumn{3}{|c|}{ Puntuación media de la PAS } \\
\hline & & Admisión & Alta & $\begin{array}{l}\text { Segui- } \\
\text { miento }\end{array}$ \\
\hline \multirow[t]{3}{*}{ Recaída } & A & 8,16 & 1,68 & 7,0 \\
\hline & $\mathrm{B}$ & 7,66 & 4,71 & 8,42 \\
\hline & A y B & 8,04 & 2,60 & 7,38 \\
\hline \multirow[t]{4}{*}{ No recaída } & C & 7,2 & 1,08 & 0,87 \\
\hline & $\mathrm{D}$ & 9,0 & 7,0 & 4,60 \\
\hline & $\mathbf{E}$ & 8,8 & 4,25 & 1,0 \\
\hline & $C, D$ y E & 7,88 & 3,09 & $1,61^{-}$ \\
\hline $\begin{array}{l}\text { Persistencia de } \\
\text { síntomas }\end{array}$ & $\mathrm{F}$ & 7,1 & 7,2 & 6,9 \\
\hline
\end{tabular}

* PAS indica Psychiatric Assessment Scala. Para una descripción de los grupos ver tabla 1.

eran jóvenes, solteros y varones, con historias psiquiátricas bien establecidas y que vivían en hogares con familiares de alta EE. Los pacientes californianos en los que no se apreciaban cambios fueron excluidos de los 
análisis subsiguientes de la relación entre las valoraciones de EE de los familiares y las recaídas, ya que no se recuperaron en ningún momento-del episodio que les llevó a ingresar.

Las medias de la PAS en severidad de delirios, alucinaciones e incoherencia para los tres momentos de evaluación para los distintos grupos pronósticos aparecen en la tabla 2.

La relación entre la rehospitalización y la recaída sintomática no fue significativa. Once ( 46 por 100 ) de los 24 pacientes que recayeron fueron reingresados. El mismo número de pacientes en el grupo de no recaída fueron reingresados (37 por 100 [11/301]). Es importante señalar que a todos los pacientes que volvieron a ingresar se les hizo de nuevo una evaluación psiquiátrica completa. Los 11 pacientes del grupo de no recaída fueron reingresados para tratamiento de depresión, manejo de desorganización social y descarga de la tensión familiar, aunque ninguno sufrió una reactivación o exacerbación de síntomas esquizofrénicos.

\section{VALORACIONES DE LA RESPUESTA EMOCIONAL}

En los estudios británicos, algo más de la mitad de las familias evaluadas fueron valoradas como bajas en EE (52 por 100 [72/138 del N combinado]), es decir, el (los) familiar (es) clave (s) hacía 5 o menos comentariós críticos, obtenía una valoración de 3 o menos en la escala de 6 puntos de implicación emocional excesiva, y no mostraba evidencias de hostilidad hacia el paciente en la entrevista. Utilizando umbrales idénticos de EE para la muestra californiana, sólo un tercio de las familias eran valoradas como bajas en $\mathrm{EE}\left(23 / 69, \mathrm{X}^{2}=6,58, \mathrm{P} \mathrm{II}<0,02\right)$. Este es uno de los resultados más sobresalientes de los obtenidos en este estudio transcultural de la EE.

La distribución de las críticas entre las dos culturas fue bastánte diferente. La impresión de los entrevistadores de que los familiares de Londres eran generalmente menos críticos, estaba avalada por los datos: el 33 por 100 de los familiares de Londres, frente a sólo un 4 por 100 de los familiares de Los Ȧngeles, no hacía ningún comentario crítico cuando hablaba del paciente. Sin embargo, el número medio de comentarios críticos hechos por los familiares ingleses y californianos era similar $(7,50 \mathrm{v} 6,86$, respectivamente; no significativo), ya que los primeros eran más extremos en su pauta de respuesta. Un pequeño número de familiares aumentaba la media de críticas en el grupo británico, con sus elevadas puntuaciones, equiparando de este modo las medias de Londres y California.

Las valoraciones de hostilidad fueron significativamente más frecuentes en la muestra de familiares californianos (28 por $100 \times 18$ por 100 , P II <0,05). En cambio, no había diferencias transculturales en la distribución de la implicación emocional excesiva. Este dato puede ser un resultado de la escasez de ejemplos de autosacrificio extremo, sobreprotección, o exceso de preocupación en ambas culturas. La mayoría de los padres no eran valorados altos en esta escala, independientemente de su origen cultural. Solamente el 21 por 100 de.los padres británicos y el 15 por 100 de los californianos fueron considerados como excesivamente implicados (es decir, obtuvieron valoraciones de 4 ó 5 , en una escala de 6 puntos). Sin embargo, las madres recibían valoraciones, significativamente mayores, en la escala de implicación emocional excesiva, que los padres (familiares californianos, $\mathrm{X}^{2}=10,42, \mathrm{P}(0,001)$; familiares londinenses, $\mathrm{X}^{2}=8.10$, $\mathrm{P}(0,002)$. 
La mejor separación en términos de tasas de recaídas se obtuvo utilizando el mismo punto de corte usado por Vaughn y Leff (6), para clasificar a las familias en el subgrupo de alta EE, es decir, un umbral de 6 comentarios críticos, o valoraciones de implicación emocional excesiva de 4 ó 5 . Todos los resultados siguientes están basados en estos umbrales de críticas e implicación emocional excesiva. La hostilidad nunca fue valorada como presente cuando un familiar hacía menos de 6 comentarios críticos, por lo tanto, no fue utilizada en la composición del grupo de alta EE.

\section{Relación entre emoción expresada y pronóstico clínico}

Estos datos fueron analizados de varias formas, todas las cuales dieron lugar a resultados similares. En primer lugar consideramos la relación entre el índice de EE de los familiares y la recaída sintomática, replicando los principales análisis de los estudios británicos. De estos análisis quedaron excluidos los 15 pacientes con alta persistencia de síntomas en los que no se apreció ningún cambio.

Los índices estadísticos sobre recaída para los 54 pacientes esquizofrénicos restantes de hogares de alta y baja $\mathrm{EE}$ aparecen en la tabla 3 . En este grupo se incluyeron 11 pacientes que durante el seguimiento vivían simultáneamente en sus hogares y en algún tipo de residencia, y 7 pacientes que no vivían en sus casas (aunque todos, menos uno, estuvieron en contacto regular con sus familiares, pasando incluso los fines de semana en casa de sus padres). Cuando se restringió el análisis de la EE-recaída al grupo nuclear de los 36 pacientes que vivían en casa continuamente, después del alta, la proporción de recaídas alcanzó los niveles que se muestran en la tabla 3. Tanto para el grupo restringido de 36 pacientes, como

\section{TABLA 3}

Relación de las valoraciones de EE de los familiares con la recaida a los nueve meses del alta*

\begin{tabular}{ccc}
\hline $\begin{array}{c}\text { Valoraciones de EE } \\
\text { de los familiares }\end{array}$ & . No Recaída & $\begin{array}{c}\text { Recaída } \\
\text { (Porcentaje) }\end{array}$ \\
\hline Alta & Muestra total, N=54** & $20(56)$ \\
\hline Baja & 16 & $3(17) \cdot$ \\
\hline & 15 & $15(60)$ \\
\hline Alta & Pacientes en casa durante el seguimiento, $\mathrm{N}=36^{* * *}$ \\
\hline Baja & 10 & $1(9)$ \\
\hline
\end{tabular}

* EE indica emoción expresada. Una valoración de alta EE era asignada cuando aparecían seis o más comentarios críticos o elevada implicación emocional (IEE). Una valoración de baja EE se otorgaba cuando los comentarios críticos iban de 0 a 5 y no había una puntuación elevada en implicación emocional excesiva (IEE).

** Yates' corrected $\mathrm{X}^{2} ; 5,92,1 \mathrm{df} ; \mathrm{y} \mathrm{P}=0,015$

*** $\mathrm{P}=0,005$ by Fisher's exact test. 
para la muestra total de 54 pacientes, se encontró una asociación significativa entre la valoración alta de $\mathrm{EE}$ y recaída $(\mathrm{N}=36, \mathrm{P}=0,009 ; \mathrm{N}=54$; $\mathrm{P}=0,015)$. La proporción de pacientes que recayeron en los grupos de alta y baja EE en ambos análisis fue similar estadísticamente a la encontrada en los dos estudios británicos (Brown et al. (5), alta EE, 58 por 100; baja $E E, 16$ por $100, P=0,001$; Vaughn y Leff $(6)$, alta EE 48 por 100 , baja $E E$ 6 por $100, \mathrm{P}=0,007)$.

Como cabía esperar, la asociación entre la valoración de EE de los familiares y la recaída fue aún más fuerte entre los pacientes que permanecieron en su casa. Era más débil, en cambio para los pacientes que vivieron fuera, al menos durante parte del seguimiento. Las tasas de recaídas para los 11 pacientes con valoraciones de alta EE y los 7 pacientes con valoraciones de baja EE en este subgrupo fueron 49 y 25 por 100 respectivamente, diferencia no significativa. El número de sujetos considerado es pequeño, pero los datos sugieren que los sujetos con valoraciones altas en EE tienen mayores probabilidades de permanecer ajustados si no están en casa continuamente, mientras sucede lo contrario para los pacientes de hogares de baja EE.

En una segunda serie de análisis, no realizados en los estudios británicos, examinamos las diferencias de intensidad de las asociaciones entre indicadores alternativos de EE (incluyendo las valoraciones de $\mathrm{EE}$ ) y medidas diferentes de pronóstico clínico para la muestra total de 69 pacientes. Estos resultados se resumen en la matriz de correlaciones incluida en la tabla 4. Los indicadores alternativos de EE fueron los siguientes: 1) el índice global empleado en el análisis de recaída-no recaída (nivel de EE); 2) la mayor puntuación de los familiares en número de críticas (ACRIT), en implicación emocional excesiva (AIEE) y hostilidad (AHOST); y 3) las puntuaciones individuales de padres y madres en estas mismas tres escalas. Las madres fueron codificadas como EE1 y los padres como EE2.

Las medidas de pronóstico clínico fueron las siguientes: 1) la puntuación total de síntomas esquizofrénicos derivada del PSE, en el seguimiento, o la recaída si ésta ocurría antes (PSE 3) (esta puntuación se compone solamente de síntomas positivos); 2) la puntuación total de síntomas de la PAS en el seguimiento, o la recaída, si aparecía con anterioridad (PAS 3) (esta puntuación está formada por síntomas positivos y negativos); y 3) una medida global de pronóstico clínico (pronóstico 2), en la que los pacientes de las caregorías de recaída y alta persistencia de síntomas fueron combinados en una categoría única de mal pronóstico. Se encontró que el nivel global de EE estaba altamente correlacionado con el pronóstico global $(\mathrm{P}<0,02)$ y con cada una de las valoraciones de EE, excepto con la implicación emocional excesiva de los padres $(\mathrm{P}<0,01)$. Todas las valoraciones en implicación emocional excesiva (de las madres, de los padres, y del familiar con mayor puntuación) mostraban correlaciones estadísticamente significativas con una o más medidas de mal pronóstico. La importancia de la valoración en implicación emocional excesiva en estos análisis fue, casi con toda certeza, debido a la gran proporción de pacientes con alta persistencia de síntomas que tenían familiares que obtenían valoraciones de 3 o más en la escala de implicación emocional excesiva (73 por 100); una proporción mucho mayor que el resto de la muestra californiana o de cualquiera de las muestras británicas. 


\section{TABLA 4}

Correlaciones entre la EE global y diferentes medidas clínicas en 69 pacientes*

\section{Eel CRIT Ee1 leE Ee1 host eE2 CRIT Ee2 IEE EE2 host PSE1 PSE2 PSE3 Nivel de Ee- ACRIT AIEE AHOST Sum PAS1 Sum PAS2 Sum PAS3 Pronóstico 2}

\begin{tabular}{|c|c|c|c|c|c|c|c|c|c|c|c|c|c|c|c|c|}
\hline EE1 CRIT ...................... 1,00 & & & & & . & ? & & & & & & & & & & \\
\hline EE 1 IEE $\ldots \ldots \ldots \ldots \ldots \ldots \ldots \ldots, \quad-0,03$ & 1,00 & & & & & & & & & & & & . & & & \\
\hline EE1 HOST ……............ $0,46^{* *}$ & $-0,23$ & 1,00 & & & & & & & & $\cdot$ & & & & & & \\
\hline 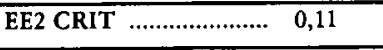 & 0,04 & 0,01 & 1,00 & & & & & & & & & & & & & \\
\hline EE2 IEE $\ldots \ldots \ldots \ldots \ldots \ldots \ldots \ldots \ldots \ldots$ & $0,30^{* *}$ & $-0,07$ & $0,30^{* *}$ & 1,00 & & & & & & & & & & & & \\
\hline EE2 HOST …................ $0,28^{* *}$ & $-0,06$ & $0,29 * *$ & $0,38 * *$ & $-0,14$ & 1,00 & & & & & & & & & & & \\
\hline PSE 1 & $-0,14$ & $0,25 * *$ & 0,06 & $\begin{array}{c}-0,13 \\
0,07\end{array}$ & 1,00 & & & & & & & & & & & \\
\hline PSE $2 \ldots \ldots \ldots \ldots \ldots \ldots \ldots \ldots \ldots \ldots$ & 0,11 & $-0,10$ & 0,02 & 0,07 & 0,001 & 0,17 & 1,00 & & & & & & & 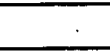 & & $\cdot$ \\
\hline 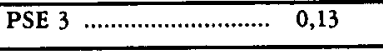 & $0,26 * *$ & $-0,11$ & 0,14 & $0,36 * *$ & 0,04 & 0,11 & 0,21 & 1,00 & & & & & & & & \\
\hline Nivel de EE ................... $0,53^{* *}$ & 0,16 & $0,25 * *$ & $0,40^{* *}$ & 0,18 & $0,39 * *$ & 0,08 & $-0,02$ & 0,20 & 1,00 & & & & & & & \\
\hline 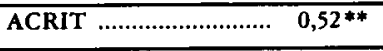 & 0,04 & 0,20 & $0,76^{* *}$ & $0,27^{* *}$ & $0,38 * *$ & 0,01 & 0,02 & 0,21 & $0,64 * *$ & 1,00 & & & . & & & \\
\hline 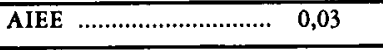 & $0,80^{* *}$ & $-0,10$ & 0,12 & $0,47^{* *}$ & 0,01 & $-0,09$ & 0,12 & $0,33 * *$ & 0,23 & 0,10 & 1,00 & & & & & \\
\hline AHOST ….................. $0,31^{* *}$ & $-0,10$ & $0,48 * *$ & $0,26 * *$ & $-0,11$ & $0,71^{* *}$ & 0,14 & $-0,05$ & 0,14 & $0,45 * *$ & $0,38 * *$ & $-0,13$ & 1,00 & & & & \\
\hline Sum PAS $1 \ldots \ldots \ldots \ldots \ldots \ldots$ & 0,08 & 0,07 & 0,05 & 0,08 & 0,14 & 0,18 & 0,10 & 0,12 & 0,17 & 0,23 & 0,19 & 0,07 & 1,00 & & & \\
\hline Sum PAS $2 \ldots \ldots \ldots \ldots \ldots \ldots . . . . . . ., 0,07$ & $0,26 * *$ & 0,03 & 0,02 & 0,12 & $-0,02$ & 0,18 & $0,57^{* *}$ & $0,25 * *$ & $-0,02$ & 0,02 & $0,27^{* *}$ & $-0,01$ & $0,43^{* *}$ & 1,00 & & \\
\hline Sum PAS $3 \ldots \ldots \ldots \ldots \ldots \ldots . . . \quad 0,09$ & $0,026^{* *}$ & $-0,02$ & 0,16 & $0,27^{* *}$ & 0,06 & 0,17 & $-0,01$ & $0,67 * *$ & $0,28 * *$ & 0,15 & $0,35^{* *}$ & 0,11 & $0,24 * *$ & $0,29 * *$ & 1,00 & \\
\hline 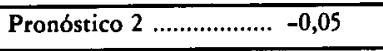 & $-0,11$ & $-0,03$ & $-0,22$ & $-0,36 * *$ & $-0,18$ & $-0,05$ & $-0,07$ & $-0,75^{* *}$ & $-0,29 * *$ & $-0,26 * *$ & $-0,21$ & $-0,22$ & $-0,11$ & $-0,12$ & $-0,63^{* *}$ & 1,00 \\
\hline
\end{tabular}

$\overline{20}$

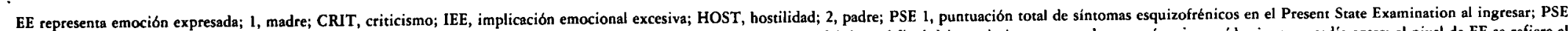

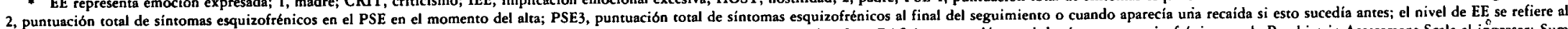

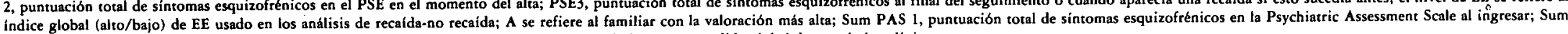
PAS 2, PAS total al alta; Sum PAS 3, puntuación total en la PAS en el seguimiento; y Pronóstico 2, una medida global de pronóstico clínico.

** Los niveles de significación para las correlaciones fueron los siguientes: $r=0,24$ a $0,31, P=0,05$ a 0,$01 ; r=0,31$ a 0,$39 ; P=0,01$ a 0,$003 ;$ y $r>0,39, P<0,003$. 


\section{FACTORES CLINICOS}

Una crítica persistente hasta la fecha, de los estudios de EE es que no excluyen satisfactoriamente la severidad del trastorno, como una explicación posible de la asociación entre la EE de los familiares y la recaída. La argumentación es la siguiente: si se observara que la alta EE correlacionara significativamente con la severidad de la psicopatología clínica del paciente en el momento de su ingreso, se podría concluir solamente que los pacientes que no funcionan bien, y con trastornos más severos, no tienen un buen pronóstico y despiertan reacciones emocionales negativas en sus familiares. Entonces, la emoción expresada sería un «artefacto» de la morbilidad y de los antecedentes de recaída, pero no su causa. Por tanto, si queremos hacer inferencias correctas respecto a la relación entre EE y pronóstico, se hace imprescindible explorar la relación entre la severidad del trastorno en el momento del ingreso y las valoraciones de los familiares en las escalas de EE.

Por consiguiente, examinamos la correlación entre el índice global de $\mathrm{EE}$ (nivel de $\mathrm{EE}$ ) y la puntuación total de síntomas esquizofrénicos en el ingreso, a partir del PSE y de la PAS, respectivamente (PSE1 y sumPAS1). Pensamos que estas puntuaciones son medidas aceptables de la severidad del trastorno. También tomamos en consideración las correlaciones entre las medidas brutas de EE en críticas, implicación emocional excesiva y hostilidad, y estos mismos índices de severidad del trastorno. Los resultados de estos análisis aparecen en la matriz de correlaciones de la tabla 4.

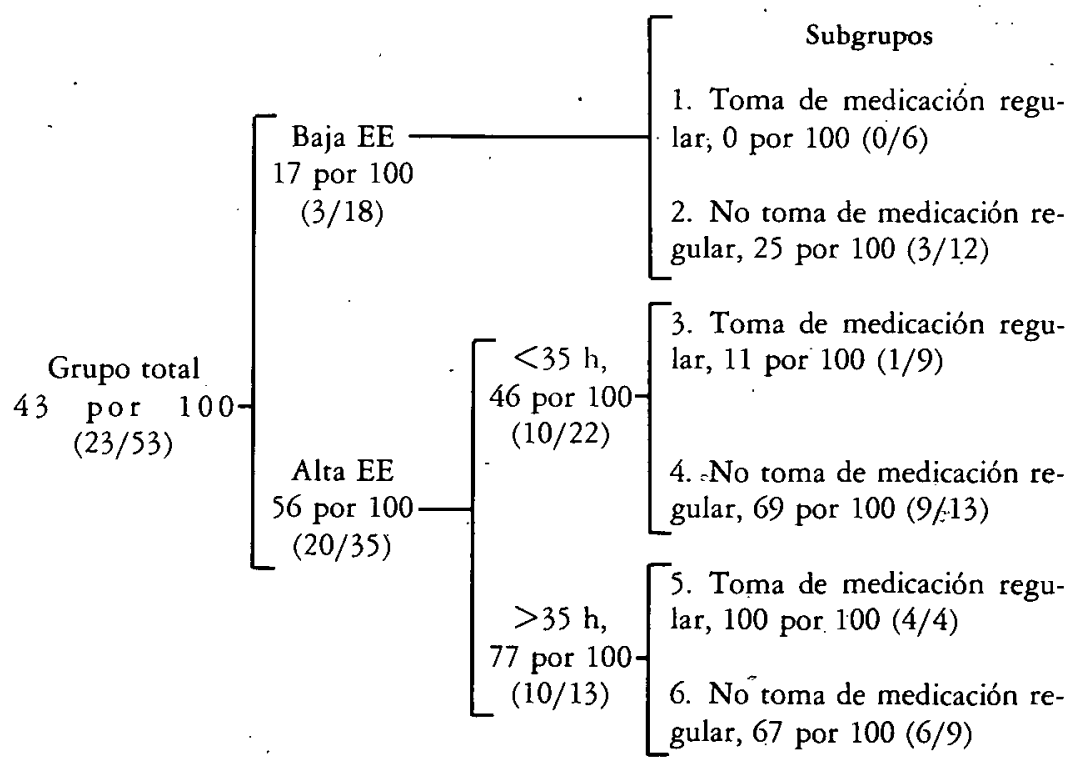

Tasas de recaída de 53 pacientes a los nueve meses. Relaciones entre las valoraciones de EE de los familiares, la medicación después del alta, el tiempo de contacto cara a cara por semana después del alta, y recaída.

No se encontraron correlaciones significativas entre el índice bajo o alto de EE (nivel de EE) y las puntuaciones, bien del PSE1 o sumPAS1. Tampoco apareció ninguna correlación significativa entre ninguna de las valoraciones de EE consideradas y la puntuación sumPAS1. En realidad, 
sólo una de las veinte correlaciones era significativa al nivel de confianza de 0,05 ; la puntuación de hostilidad de las madres estaba correlacionada con la puntuación del PSE1. Dado el número de correlaciones, este único resultado significativo puede ser debido al azar.

La matriz también revela la falta de asociación entre las medidas de severidad del trastorno al ingreso y las tres medidas de pronóstico utilizadas.

Las correlaciones entre las valoraciones de EE de los familiares, la severidad del trastorno, y el pronóstico clínico representados en la matriz de la tabla 4, sugieren claramente que la EE no es un artefacto de la morbilidad del paciente. Su asociación con la recaída, y con un mal pronóstico, no puede ser explicada por la condición clínica del paciente al ingresar.

Además de los análisis precedentes, se llevaron a cabo análisis de $\mathrm{X}^{2}$ entre los síntomas elicitados por el PSE y la PAS para determinar si la presencia de determinados síntomas en el ingreso o en el alta estaban relacionados con el pronóstico a los nueve meses, en el grupo total de seguimiento. Los análisis de $\mathrm{X}^{2}$ fueron también realizados para los 140 síntomas identificados por el PSE en el momento del ingreso, para determinar si la presencia de alguno de ellos estaba relacionada con las valoraciones de EE de los familiares. Ningún ítem estaba significativamente relacionado con el pronóstico, y no existían diferencias en la presencia o ausencia de síntomas específicos entre los pacientes de hogares de alta y de baja EE. Además, una proporción equivalente de pacientes con valoraciones de alta y de baja EE presentaban remisión de síntomas en el momento del alta.

Ninguna otra variable clínica, ni ninguna otra.característica de la historia psiquiátrica deł paciente (por ej., el status del paciente en el ingreso, el inicio agudo, el tiempo transcurrido desde la primera aparición del trastorno), añadieron valor alguno al índice de EE en la predicción de una recaída. Este resultado concuerda exactamente con los hallazgos británicos.

\section{FACTORES SOCIODEMOGRAFICOS}

En los estudios británicos, dos factores demográficos estaban fuertemente relacionados con la recaída sintomática; sexo y estado civil. En el presente estudio, el estado civil no pudo ser considerado, ya que sólo 4 de los 54 pacientes estaban casados y vivían con sus cónyuges al ser ingresados. Sin embargo, la diferencia de sexo parece ser importante. Como en los estudios británicos, había una relación significativa entre sexo y pronóstico $[\mathrm{P}<0,05]$; la tasa de recaída para hombres era más del triple que para mujeres. Además, los sujetos varones con valoraciones de alta EE fueron los responsables de esta fuerte asociación, como indican las siguientes tasas de recaída. Pará sujetos varones, los que tenían valoraciones de alta EE, tuvieron una tasa de recaídas del 66 por 100 (19/29), aquellos con bajas valoraciones de EE presentan una tasa del 17 por $100(2 / 12)$; y la tasa global fue del 51 por 100. En el caso de las mujeres, las de alta EE recayeron en un 14 por $100(1 / 7)$ y las de baja EE en un 17 por $100(1 / 6)$; la tasa global fue del 15 por 100 .

Los dos estudios británicos descubrieron diferencias similares respecto al sexo. Los datos británicos sugieren dos razones relacionadas para explicar la gran diferencia entre hombres y mujeres. Ambas recibieron apoyo del análisis de los datos del estudio de California del Sur. En primer lugar, los pacientes varones solteros de hogares de alta EE tenían significativa- 
mente menos probabilidades que sus equivalentes mujeres de estar protegidos tanto por la administración regular de medicación como por el bajo contacto con los familiares (California, $\mathrm{P}=0,004$; Londres, $\mathrm{P}=0,006$ ). La influencia de estos dos factores protectores es revisada en detalle en la próxima sección. En segundo lugar, el conflicto parental, que se ha mostrado asociado con una alta tasa de recaídas en los pacientes solteros $(\mathrm{P}<0,05$, estudios de California y Londres), estaba presente con más frecuencia en los hogares con ambos padres donde el paciente era hijo que en aquellos casos que el paciente era hija ( $\mathrm{P}<0,05$ en ambos estudios). Por tanto, los pacientes solteros, que quizá necesiten de una mayor protección de los efec-

TABLA 5

Relaciones entre la valoración de los familiares (EE), medicación después del alta, y recaída

\begin{tabular}{|c|c|c|c|c|}
\hline \multirow{3}{*}{$\begin{array}{c}\text { EE de los } \\
\text { familiares* }\end{array}$} & \multicolumn{4}{|c|}{ ADMINISTRACION DE LA MEDICACION } \\
\hline & \multicolumn{2}{|c|}{ IRREGULAR } & \multicolumn{2}{|c|}{ REGULAR } \\
\hline & No Recaída & $\begin{array}{c}\text { Recaida } \\
\text { (Porcentaje) }\end{array}$ & No Recaída & $\begin{array}{c}\text { Recaida } \\
\text { (Porcentaje) }\end{array}$ \\
\hline & \multicolumn{4}{|c|}{ Muestra total, $\mathrm{N}=53 * *$} \\
\hline Alta & 7 & $15(68)$ & 8 & $5(38)$ \\
\hline 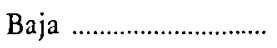 & 9 & $3(25)$ & 6 & $0(0)$ \\
\hline TOTAL ............. & 16 & $18(53)$. & 14 & $5(26)$ \\
\hline
\end{tabular}

Pacientes en casa durante el seguimiento, $\mathrm{N}=35 * * *$

\begin{tabular}{cccccc} 
Alta ........................ & 4 & $11(73)$ & 5 & $4(44)$ \\
\hline Baja ........................ & 7 & $1(12,5)$ & 3 & $0(0)$ \\
\hline Total ........... & 11 & $12(52)$ & 8 & $4(33)$ \\
\hline
\end{tabular}

Pacientes que no estaban en casa durante el seguimiento, $\mathrm{N}=18 * * * *$

\begin{tabular}{|c|c|c|c|c|}
\hline Alta & 3 & $4(57)$ & 3 & $1(25)$ \\
\hline 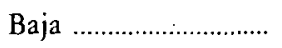 & 2 & $2(50)$ & 3 & $0(0)$ \\
\hline 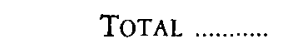 & 5 & $6(56)$ & 6 & $1(14)$ \\
\hline
\end{tabular}

* EE indica emoción expresada. Los análisis de $\mathrm{X}^{2}$ sobre medicación regular/irregular no fueron significativos en ninguno de los grupos.

** El test exacto de Fisher, $\mathrm{P}=0,019$ para el grupo de alta EE-medicación irregularrecaída / grupo de baja EE-medicación irregular-recaída, y para el grupo de alta EE-medicación regular-no recaída / grupo de recaída, no fue significativo.

*** El test exacto de Fisher, $\mathrm{P}=0,008$ para el grupo baja EE-medicación irregular-recaída / grupo de alta EE-medicación irregular-recaída, y para el grupo de baja EE-no recaída / alta EE-medicación regular-recaida, no fue significativo. Se obviaron los datos de un paciente.

**** El test exacto de Fisher no fue significativo para ninguna de las comparaciones entre grupos. 
tos de un ambiente estresante, eran los que tenían menos posibilidad de recibirla.

Una variable final que interactuaba con el sexo y el pronóstico, solamente para los pacientes de California, fue el uso de drogas (exceptuandó la marihuana). El abuso de drogas entre los pacientes británicos era raro, y servía como motivo de exclusión de los estudios de EE. En el estudio de California, a causa del número de pacientes afectados, los casos sólo eran excluidos cuando el diagnóstico principal de esquizofrenia era dudoso, por un abuso de drogas reciente. El 44 por 100 de la muestra de 54 pacientes había usado drogas como cocaína, barbitúricos y «polvo de ángel» en el año anterior al ingreso. Los pacientes que informaban de abuso de drogas tenían una probabilidad significativamente mayor de recaer que los que no las consumían (P) 0,05. Los consumidores de droga tendían a ser varones, de hogares de alta EE y no tomaban medicación regularmente, aunque ninguna de estas tendencias era estadísticamente significativa.

\section{FACTORES POSTERIORES AL ALTA: MEDICACION ANTIPSICOTICA Y CONTACTO FAMILIAR}

En los estudios británicos, la terapia de mantenimiento con fenotiacinas y el contacto reducido con los familiares (menos de 35 horas por semana) mejoraban el pronóstico de los pacientes de hogares de alta EE. Ningún factor alcanzó significación para el grupo de baja EE durante el período de seguimiento de nueve meses: era probable que estos pacientes permanecieran bien, independientemente de las pautas de administración de la medicación o del contacto familiar. Sin embargo, un seguimiento de dos años de los pacientes del estudio de Vaughn y Leff de 1976 reveló un efecto preventivo significativo de la medicación en el grupo de baja EE durante este período de tiempo más largo (7). Es destacable que ningún paciente con una valoración de baja EE del estudio de 1976 tuvo una recaída mientras tomó la medicación con regularidad en los dos años después del alta. Aunque, extraordinariamente similares a los resultados británicos, los resultados californianos respecto al papel de la medicación en la prevẹnción de recaídas deben ser tomados con una cierta cautela, ya que las condiciones para evaluar la colaboración de los pacientes en los regímenes de medicación prescritos eran menos satisfactorias. Prácticamente, todos los pacientes británicos asistían regularmente a una clínica ambulatoria después de sus altas hospitalarias. Las notas en el historial psiquiátrico se hacían en intervalos de 3 ó 4 semanas, consiguiendo una información detallada sobre los cambios en la medicación o de las pautas de ingestión, independientemente de los informes del paciente durante el seguimiento. Cualquier duda sobre la medicación podía ser abordada con el clínico responsable gracias a los vínculos naturales entre el hospital, la clínica ambulatoria y la unidad de investigación.

Por el contrario, pocos pacientes del estudio de California recibieron algun tipo de cuidado poshospitalario. Solamente el 36 por 100 de la muestra total cumplía satisfactoriamente los siguientes criterios de terapia de mantenimiento regular: uso de dosis prescritas de neurolépticos durante al menos el 75 por 100 del período de nueve meses de seguimiento, con ningún período de cuatro semanas consecutivas sin tomar medicación. Esta fue una proporción menor que en los dos estudios británicos. Proporciones equivalentes de pacientes con valoraciones de alta y de baja EE dejaron de tomar la medicación regularmente. Solamente 3 de los 25 pacientes 
que se consideraba que tomaban medicación regular recibieron inyecciones de neurolépticos de larga acción. Los juicios sobre las pautas de toma de medicación se basaban principalmente en preguntas específicas al paciente, por parte del psiquiatra de la investigación durante el seguimiento, complementadas con la información de las llamadas telefónicas mensuales a la familia. A falta de contacto regular con los responsables clínicos, el psiquiatra investigador intentaba ser lo más riguroso posible en su evaluación, procuraba tomar todo tipo de precauciones. Si existía cualquier duda sobre la regularidad de las tomas, se consideraba que el paciente no tomaba la medicación regularmente.

Los análisis de la relación entre la valoración de la EE de los familiares y la medicación del paciente y la recaída produjeron los resultados mostrados en la tabla 5 . Los valores de baja EE fueron muy similares a los de los pacientes británicos con puntuaciones de baja EE: las tasas de recaída a los nueve meses eran bajas, tomaran o no la medicación regularmente. Realmente, los valores de medicación mostrados en la tabla 5 parecen apoyar una de las primeras interpretaciones de los resultados británicos: que la medicación antipsicótica no es necesaria para pacientes con valoraciones de baja EE. Pero hay evidencias que contradicen esta interpretación. El subgrupo de pacientes californianos con valoraciones de baja $\mathrm{EE}$ que vivían fuera de sus hogares durante el seguimiento no se mantuvo tan bien como el grupo que permaneció en sus casas, en ausencia de medicación regular, tal como aparece en la tabla 5 . A pesar de que se trata de números pequeños de sujetos, es posible especular que, cuando los pacientes de baja EE están fuera de un ambiente familiar que les apoya, tienen un mayor riesgo de recaída, quizá debido a que puedan verse sometidos a una mayor presencia de acontecimientos vitales (16). En estas ocasiones se pueden beneficiar de la terapia de mantenimiento. Finalmente, es impresionante que en este estudio, como en el británico de 1976, ningún paciente de baja EE que estuviera tomando la medicación regularmente tuvo una recaída durante el seguimiento. Por el contrario, la tasa de recaídas entre los pacientes de alta $\mathrm{EE}$ que tomaban medicación era alta, alcanzando un 44 por 100 en el grupo de los pacientes que permanecieron en sus hogares. Aunque las pruebas de $\mathrm{X}^{2}$ revelaron un efecto independiente $\mathrm{e}$ inconfundible de la medicación $\left(X^{2}=4,77, P=0,290\right)$, los regímenes de medicación regular parecían ofrecer a los pacientes de hogares de alta EE solamente una protección limitada contra la recaída.

La asociación entre nivel de contacto con los familiares y las recaídas en los pacientes de los hogares de alta EE no alcanzó significación estadística, aunque la relación era en la dirección esperada. Los pacientes con valoraciones de alta EE tenían una mayor tasa de recaídas (77 por 100) si veían a sus familiares más de 35 horas a la semana, pero la tasa de recaídas del 46 por 100 en el grupo de bajo contacto era mayor que en los dos grupos semejantes de los estudios británicos. Al mismo tiempo, las proporciones de baja EE, de nuevo, eran muy similares a las equivalentes británicas: las pautas de contacto entre los pacientes con valoraciones de baja EE y sus familiares no producen ninguna diferencia en sus tasas de recaídas. La figura resume los efectos aditivos de los tres factores: la emoción expresada de los familiares, la medicación, y el contacto directo cara a cara. Este análisis se llevó a cabo con la muestra total de 54 pacientes, menos uno en el que faltaba la información sobre el contacto y la medicación. La tasa de recaídas correspondiente al grupo que permaneció en su casa $(\mathrm{N}=35)$ fue muy similar.

El análisis reveló una influencia considerable de la combinación de la 
medicación regular y el bajo contacto sobre las pautas de recaída de los pacientes de alta EE. Las tasas de recaída de los subgrupos indicaron que el mejor pronóstico para los pacientes con valoraciones de alta EE se da cuando están protegidos por el bajo contacto con sus familiares y por la terapia regular de mantenimiento simultáneamente (subgrupo 3). La tasa de recaída para estos pacientes fue de un 11 por 100 en la muestra total, y del 0 por 100 en el grupo de pacientes que permanecieron en casa. Estas tasas fueron comparables a las de los pacientes de baja EE (subgrupos 1 y 2), y significativamente menores que las de los pacientes de alta $\mathbf{E E}$, en los que ningún factor protector estaba actuando (subgrupo $6 ; \mathrm{N}=53 ; \mathrm{P}=0,25$; $\mathrm{N}=35 ; \mathrm{P}=0,28$ ). Este resultado replicaba exactamente los principales resultados de un análisis similar realizado para los 128 pacientes británicos de los estudios de Londres de 1972 y 1976 (6). Sin embargo, algunos resultados del estudio californiano difieren de los datos del estudio británico, en la medida que sugieren que la medicación ofrece protección contra la recaída a los pacientes de alta $E E$, solamente si además ven a sus familiares críticos o con excesiva implicación emocional, menos de 35 horas a la semana. Si sólo actúa uno de los factores protectores (subgrupos 4 y 5) las tasas de recaída se mantienen altas. En realidad, la mayor tasa de recaída en el estudio (100 por 100) aparecía en los pacientes que vivían en hogares de alta $\mathrm{EE}$, que tomaban medicación regularmente, pero que pasaban más de 35 horas a la semana con sus familiares.

Por tanto, en los pacientes del estudio californiano (a diferencia de sus equivalentes británicos), la medicación regular y el contacto reducido se comportaban como factores interactivos, y no aditivos. Los datos indican que para que los pacientes de alta EE eviten las recaídas son necesarios ambos factores.

\section{Resumen de los factores relacionados con la recaída}

El sexo masculino, el conflicto parental (en hogares con ambos padres) y la ausencia de medicación regular estaban asociados con una alta probabilidad de recaída en el estudio de California, al igual que en los estudios británicos. Una cuarta variable, el abuso de drogas, estaba también asociada con la recaída, pero sólo en nuestro estudio. En alguna medida, estos factores pueden estar confundidos con'otros, y serían necesarias muestras mayores y con una representación demográfica más equilibrada que la nuestra, para determinar satisfactoriamente sus efectos independientes. Sin embargo, para cada variable de riesgo, la presencia de un farniliar de baja EE tenía una influencia protectora, y cuando la variable fue controlada, se seguía manteniendo la relación principal entre EE y recaída. Por lo tanto, el test de $\mathrm{X}^{2}$ mostró un efecto inconfundible de la emoción expresada sobre la recaída, independientemente de la medicación $\left(X^{2}=9,66\right.$; $\mathrm{P}=0,0019$ ).

\section{COMENTARIO}

Este intento transcultural de replicar los resultados británicos en relación con la influencia de la familia sobre el curso de la esquizofrenia ha -alcanzado resultados positivos, a pesar de las diferencias de las muestras de pacientes que hemos mencionado. La asociación entre las valoraciones de EE de los familiares y las recaídas de los pacientes se ha mantenido en 
el estudio americano. Esta constituye la tercera réplica que llega al resultado de que un alto número de críticas, un alto nivel de implicación emocional excesiva, expresado por un familiar, es el mejor predictor aislado de la aparición o exacerbación de síntomas esquizofrénicos positivos durante los nueve meses siguientes al alta. Esta relación de nuevo es independiente de todas las otras variables evaluadas, como la severidad del trastorno al ingreso, la condición clínica al alta y distintos aspectos de la historia psiquiátrica. Es posible, no obstante, que alguna variable interviniente (aún no identificada) pueda explicar o mediatizar la relación entre EE y recaída. Las interpretaciones causales solamente pueden ser confirmadas a través de estudios de intervención, en los que los esfuerzos clínicos para manipular experimentalmente la EE de los familiares o para disminuir la «temperatura emocional» en el hogar produzcan un cambio en la tasa de recaídas en la dirección prevista. Se han iniciado varios programas de estas características, logrando unos resultados iniciales prometedores (17-21).

Había algunas diferencias notables entre los sujetos de California y de los de Londres, la principal de ellas en relación con las pautas de EE de los familiares de las dos culturas. En Londres, la mayoría de las familias evaluadas fueron consideradas de baja EE, mientras que en la muestra californiana sólo un tercio de la muestra recibió esta consideración. Las valoraciones de hostilidad también fueron significativamente más frecuentes en California. Estas diferencias transculturales en el estilo de respuesta no pudieron atribuirse a los mayores niveles de deterioro y desorganización presentes en la muestra californiana, ya que no estaban relacionadas con el nivel de EE de los familiares en este estudio. Los pacientes de hogares de baja EE presentaban iğual nivel de deterioro y. de desorganización que los pacientes de hogares de alta EE, según cualquiera de los criterios utilizados. Por tanto, las diferencias en la distribución de alta y baja EE en los dos centros siguen sin ser explicadas. Sean cuales fueran las razones de estas diferencias, lo importante es que el estilo de respuesta de alta EE ha mantenido su poder predictivo en diferentes culturas, utilizando los mismos puntos de corte.

Esta experiencia de réplica transcultural ha esclarecido algunos de los problemas generalmente encontrados en los estudios de réplica. A pesar de nuestra intención inicial de realizar una repetición lo más exacta posible de los métodos y procedimientos británicos fue necesario introducir algunas modificaciones, a causa de las diferencias inevitables entre los centros de Los Angeles y Londres. Entre ellas figuran las diferencias de las poblaciones de pacientes, el lugar de investigación, la duración de las hospitalizaciones y los sistemas de atención poshospitalarios. De fracasar en replicar los resultados británicós, deberíamos dirigir nuestra atención, antes de nada, a estas diferencias, a la hora de dar posibles explicaciones sobre los resultados discrepantes.

El enfoque del MHCRC de las recaídas esquizofrénicas representa una serie de avances metodológicos. Se diferencia del usado en los estudios británicos, en las valoraciones de la condición clínica en el alta y el minucioso chequeo telefónico mensual. Se distingue de otros muchos estudios en su intento de operacionalizar las recaídas, cuantificando los síntomas. El interés de este estudio, así como de los estudios británicos, se ha centrado exclusivamente en la reaparición o la exacerbación de los síntomas esquizofrénicos positivos, dejando de lado otras medidas pronósticas, que no dejan de ser igualmente importantes. Aunque tanto los resultados de California como los de Londres sugieren que vivir en un hogar de baja EE está asociado con una menor susceptibilidad a la recaída con síntomas floridos, 
esto no implica necesariamente que el paciente esté bien. Se hace necesario prestar mayor atención a otros índices de éxito o fracaso en la comunidad, como por ejemplo el ajuste social y laboral y la calidad general de vida del paciente. También es importante evaluar dimensiones relevantes para determinar el impacto de la EE en estos diferentes pronósticos, tanto en ambientes familiares como de otra clase.

A lo largo de esta réplica se llevaron a cabo muchas comparaciones transculturales; muchos de los análisis realizados produjeron resultados muy similares a los obtenidos en los estudios británicos. Los resultados de mayor interés, quizá, son los relacionados con el papel de la medicación en la prevención de recaídas. Se ha confirmado la importancia de la medicación como un factor protector, particularmente para los pacientes esquizofrénicos con mayor riesgo de recaída, por los ambiẹtes estresantes de alta EE. No obstante, los resultados del estudio californiano difieren de los británicos, en la indicación de que la medicación proporciona protección contra la recaída, a los pacientes de alta $\mathrm{EE}$, solamente si también existe un bajo nivel de contacto $(<35 \mathrm{~h} / \mathrm{sem})$ con los familiares críticos o excesivamente implicados. En Londres se redujo la tasa de recaídas de alta EE si actuaba al menos uno de los dos factores protectores. Este fenómeno no se observó en California; la tasa de recaídas de alta EE permaneció elevada, a menos que ambos factores actuaran simultáneamente. Este resultado puede quedar explicado por la mayor proporción de pacientes varones solteros con trastornos crónicos en la muestra californiana. Conforme a la evidencia de todos los estudios de EE, los pacientes con indicadores de pronóstico menos favorables eran los que necesitaban un máximo de protección, que podía ser conśeguida a través dé 1) un ambiente de mayor neutralidad emocional, de un hogar de baja EE, o 2) los efectos combinados de la medicación regular y el contacto reducido con los familiares de alta EE.

Uno de los puntos débiles de este estudio era que las condiciones para determinar si los pacientes tomaban medicación regularmente eran menos favorables que en los dos estudios británicos. Aunque ningún estudio natural puede replicar las condiciones óptimas de un estudio controlado, los investigadores británicos consiguieron, razonablemente, designar con fiabilidad la toma regular o irregular de medicación, gracias a los cuidados poshospitalarios rutinarios of recidos a sus pacientes. En comparación, los pacientes californianos recibieron un menor nivel de tratamiento. La tónica general de cuidados recibidos varió ampliamente, y muy pocos pacientes que tomaban medicación regularmente la recibieron en condiciones óptimas. No obstante, las tasas de recaídas de los pacientes californianos que tomaban medicación y que no la tomaban fueron similares a las obtenidas en los estudios británicos. También fueron consistentes con los resultados de estudios controlados recientes con fármacos, que mostraron una tasa notable de recaídas en pacientes con medicación (22), aun cuando la toma quedaba garantizada por la administración parenteral $(23,24)$. Hogarty et al. (24) encontraron que el predictor más significativo de recaídas en pacientes que recibieron decanoato de flufenacina fue la cantidad de estrés intrafamiliar en el hogar, antes del tratamiento.

Considerados conjuntamente, los resultados sobre la medicación de los tres estudios de EE tienen implicaciones importantes para el tratamiento de la esquizofrenia. Para los casos en que los pacientes viven con los familiares, los resultados de Londres y de California sugieren que los clínicos deberían remitirse hacia la atmósfera emocional del hogar para explicar los fracasos de la medicación. Ningún paciente de baja EE que estu- 
viera tomando medicación regularmente recayó durante el período de seguimiento de nueve meses de este estudio, o durante un período de seguimiento de dos años en el estudio de Londres de 1976 (7). Sin embargo, en ambos estudios, una minoría significativa de pacientes de alta EE ( 44 por 100) recayó mientras tomaba medicación. Como casi siempre, estos pacientes que recayeron eran varones y solteros, y tenían un alto contacto con sus familiares; cuando la madre y el padre del paciente vivían juntos, era común el conflicto parental. La vulnerabilidad especial de estos pacientes reclama un examen minucioso, que queda fuera del alcance de nuestro informe. Pero los datos de los estudios de California y Londres sugieren que con la actuación aislada de las terapias de mantenimiento, es improbable que se neutralicen los efectos de tales ambientes familiares negativos. Para que estos pacientes más vulnerables puedan mantenerse ajustados en la comunidad durante mayor tiempo, después del alta, serán necesarios, casi con toda certeza, intervenciones familiares directas.

Las intervenciones familiares pueden adoptar diversas formas. Por ejemplo, pacientes y familiares pueden aprender mejores métodos de enfrentamiento con el estrés, aumentando su comprensión del trastorno y recibiendo entrenamiento y,en métodos conductuales de resolución de problemas. Los resultados a los nueve meses, de un estudio controlado, que utilizó un enfoque de este tipo en los hogares de los pacientes, reveló su superioridad sobre medidas de intervención de tipo individual, en la prevención de las principales exacerbaciones sintomáticas (19). Los resultados de esta intervención familiar y de otras basadas en la EE sugieren que tanto pacientes como familiares se benefician de intentos sensibles de informar y aconsejar: los sentimientos de vergüenza y. autoculpabilización consiguen ser disminuidos.

Pero ¿qué ocurre con los pacientes esquizofrénicos excluidos de este estudio por no vivir con sus familiares? El dato de que los pacientes de nuestro estudio representan menos del 20 por 100 de los pacientes esquizofrénicos ingresados en el Hospital Estatal de Camarillo durante el período considerado plantea una importante cuestión sobre la relevancia de la investigación de la EE para los pacientes no angloamericanos o que no viven con sus otros significativos. Desde nuestro punto de vista, la EE de los familiares representa un tipo de estrés ambiental, y los resultados en relación con su impacto sobre las pautas de recaída permiten hacer predicciones acerca del posible impacto de otros estresores de características similares, en los pacientes sin vínculos familiares. Si algunas personas tienen tendencia a sufrir episodios de esquizofrenia, a causa de algún tipo de vulnerabilidad psicobiológica subyacente, y por tanto dificultades relacionadas en el procesamiento de estímulos complejos y el manejo de las relaciones sociales, es probable que se encuentren con distintos tipos de ambiente que les creen dificultades, como residencias y centros protegidos, el puesto de trabajo o determinados ambientes terapéuticos. Queda para otros investigadores explicar las considerables tasas de recaídas (aun recibiendo dosis óptimas de medicación) de pacientes esquizofrénicos que no viven con sus familias. Nosotros tenderíamos a pensar, no obstante, que los ambientes a los que estos pacientes regresan después del alta poseen al menos algunas de las respuestas. 


\section{REFERENCIAS}

(1) LEFF, J. P. «Schizophrenia and sensivility to the family environment». Schizophrenia Bulletin, 2, 1976, 566-574.

(2) Brown, G. W.; Carstairs, G. M., y Topping, G. G. «The post-hospital adjustment of chronic mental patients». Lancet, 1958, 2, 685-689.

(3) Brown, G. W. «Experiences of discharged chronic schizophrenic mental hospital patients in various types of living group». Milbank Memory Foundation Quarterly, 1959, 37, 105-131.

(4) Brown, G. W.; MONCK, E. M.; CARstairs, G. M., Y WING, J. K. «Influence of family life on the course of schizophrenic illness». British Journal of Preventive and Social Medicine, 1962, 16, 55-68.

(5) BRown, G. W., BIRLEY, J. L. T., y WING, J. K. "Influence of family life on the course of schizophrenic disorders: a replication». British Journal of Psychiatry, 1972, 121, 241-258.

(6) VAuGHN, C. E.; y LEFF, J. P. «The influence of family and social factors on the course of psychiatric illness: a comparison of schizophrenic and depressed neurotic patients». British Journal of Psychiatry, 1976, 129, $125-137$.

(7) LeFF, J. P., y VAUGHN, C E. «The role of maintenance therapy and relatives expressed emotion in relapse os schizophrenia; a two-year follow-up». British Journal of Psycbiatry, 1981, 139, 102-104.

(8) Tarrier, N.; Vaughn, C.; Lader, M. H., y LeFF, J. P. «Bodily reactions to people and events in schizophrenia». Archives of General Psychiatry, 1979, 36, 311-315.

(9) Sturgeon, D.; Kuipers, L.; Berkowitz, R.; TurPIN, G., y LefF, J. P. «Psychofysiological responses of schir zophrenic patients to high and low expressed emotion relatives». British Journal of Psychiatry, 1981, 138, 40-45.

(10) WING, J. K.; COOPER, J. E., y SARTORIUS, N. The Description and Classification of Psychiatric Symptoms: An Instruction Manual for the PSE and Catego System. Cambridge, England, Cambridge University Press, 1974.

(11) Krawiecka, M.; Goldberg, D., y Vaughan, M. «A standardized psychiatriassessment scale for rating chronic psychotic patients». Acta Psychiatrica Scandinavica, 1977, 55, 299-308.

(12) VAUGHN, C. E., y LEFF, J. P. «The measurement of expressed emotion in the families of psychiatric patients». British Journal of Social and Clinical Psychology, 1976, 15, 157-165.

(13) BRown. G. W., y RUTTER, M. «The measurement of family activities and relationships: a metodological study». Human Relations, 1966, 19, $241-26$.

(14) RUtTer, M., y BROWN, G. W. «The reliability and validity of measures of family life and relationships in families containing a psychiatric patient». Social Psychiatry, 1966, 1, 38-53.

(15) KUIPERS, L. «Expressed emotion: a review». British Journal of Social and Clinical Psycbology, 1979, 18, 237-243.

(16) LeFF, J. P., y VAughN, C. E. «The interaction of life events and relatives' expressed emotion in schizophrenia and depressive neurosis». British Journal of Psychiatry, 1980, 136, 146-153.

(17) Anderson, C. M.; Hogarty, G. E., y Reiss, D. J. "Family treatment of adult schizophrenic patients: a psychoeducational approach». Schizophrenia Bulletin, 1980, 6, 490-505.

(18) Falloon, I. R. H.; Liberman, R. P.; Lillie, F. J., y Vaughn, C. E. «Family therapy of schizophrenics with high risk of relapse». Family Process, 1981, 20, 211-221.

(19) Falloon, I. R. H.; Boyd, J. L.; MCGill, C. W.; Razani, J.; Moss, H. B., y Gilderman, A. H. (1982). «Family management in the prevention of exacerbation of schizophrenia: a controlled study". New England Journal of Medicine, 1982, 306, 1437-1440.

(20) LefF, J.; Kuipers, L.; Berkowitz, R.; Eberlein-VRies, R., y Sturgeon, D. «A controlled trial of social intervention in the families of schizophrenic patients». British Journal of Psychiatry, 1982, 141, 121-134.

(21) Liberman, R. P.; Wallace, C. J.; Vaughn, C. E.; SNYder, K. S., y Rust, C. «Social and family factors in the course of schizophrenia: towards an interpersonal problem-solving therapy for schizophrenics and their families", en J. S. Strauss, M. Bowers, T. W. Downey et al. (eds.): The Psychotberapy of Schizophrenia. Nueva York, Plenum Publishing Corporation, 1980, 21-54.

(22) JoHnSON, D. A. W. «The duration of maintenance therapy in chronic schizophrenia». Acta Psychiatrica Scandinavica, 1976, 53, 298-301.

(23) Falloon, I.; WATT, D. C., y SHEPHERD, M. «A comparative controlled trial of pimozide and fluphenazine decanoate in the continuation therapy of schizophrenia». Psychological Medicine, 1978, 8, 59-70.

(24) Hogarty, G. E.; Schooler, N. R.; Ulrich, R.; Mussare, F.; Ferro, P. y Herron, E. «Fluphenazine and social therapy in the aftercare of schizophrenic patients: relapse analyses of a two-year controlled study of fluphenazine decanoate and fluphenazine hydrochloride». Archives of General Psychiatry, 1979, 36, 1283-1294. 